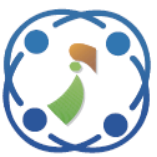

\title{
Low-Complexity based TDE Method for OFDM Signal in Higher Time-Varying Fading Channels
}

\author{
Saha Tanangsanakool $^{1} \quad$ Pongsathorn Reangsuntea $^{2} \quad$ Kazuo Mori $^{2} \quad$ Pisit Boonsrimuang $^{1 *}$ \\ ${ }^{1}$ Department of Telecommunication Engineering, Faculty of Engineering, \\ King Mongkut's Institute of Technology Ladkrabang, Bangkok, 10520 Thailand \\ ${ }^{2}$ Department of Electrical and Electronic Engineering, Graduate School of Engineering, \\ Mie University, Mie, 514-8507 Japan \\ * Corresponding author's Email: kbpisit@kmitl.ac.th
}

\begin{abstract}
Orthogonal Frequency Division Multiplexing (OFDM) signal would be damaged significantly by intercarrier interference (ICI) in higher time-varying fading channels. The ICI leads to fatal degradation of bit error rate (BER) performance due to the loss of orthogonality among subcarriers. To solve this problem, this paper proposes a high accuracy time-domain channel impulse response (CIR) estimation method and low-complexity based timedomain equalization (TDE) method for solving the simultaneous equations instead of using an inverse matrix calculation which can achieve better BER performance and lower computation complexity even in higher time-varying fading channels. The salient features of proposed method are to employ a time-domain training sequence (TS) in the estimation of channel impulse response (CIR) instead of using pilot subcarriers in the frequency domain and to employ the time domain equalization (TDE) method with maximum likelihood (ML) estimation instead of using a conventional frequency domain equalization (FDE) method. This paper also proposes a low-complexity iterative method for solving the simultaneous equations instead of using an inverse matrix calculation, which remains the computation complexity up to $7.8 \%$ of inverse matrix calculation with the same BER performance but achieves the BER performance when compared with the conventional method. This paper presents various simulation results in higher time-varying fading channels (vehicle speed $\approx 381 \mathrm{~km} / \mathrm{hrs}$ ) to demonstrate the effectiveness of the proposed method as compared with conventional FDE and TDE methods.
\end{abstract}

Keywords: OFDM, ICI, CIR, FDE, TDE, Maximum likelihood (ML) Estimation, Training sequence (TS).

\section{Introduction}

Orthogonal frequency division multiplexing (OFDM) technique has been received very much attention especially in the field of wireless communications because of its efficient usage of frequency bandwidth and robustness to multipath fading channels. From these advantages, OFDM already adopted as standard transmission techniques in various wireless communications systems such as digital video broadcasting (DVB), wireless local area network (WLAN), and fourth-generation (4G) cellular systems [1-5].

The frequency-domain equalization (FDE) method is usually employed to compensate for the fading distortion in the OFDM systems under the quasi-static multipath fading channels, where channel impulse response (CIR) in the time domain can be considered as a constant during one OFDM symbol period. In other words, the channel frequency response (CFR) which be used in the conventional FDE method and assumes constant during one OFDM symbol period. However, the CIR would be no more constant even during one OFDM symbol period in higher time-varying fading channels and causes the loss of orthogonality among subcarriers due to inter-carrier interference (ICI) [6-8]. From this fact, it is hard to compensate for the ICI by using the conventional FDE method, and the bit error rate (BER) performance of the OFDM signal would be degraded relatively in higher time-varying fading channels.

To solve the above problems, many authors have contributed many equalization methods to combat the ICI and also many methods are employed the 
estimated CIR at every sampling time to compensate for the ICI [9-13]. In [9] and [10], the equalization method is operated in the frequency domain. These would lead to higher computational complexity due to the reconstruction of the inverse matrix. In [11] and [12], the ICI mitigation methods were proposed in the time domain, however, both methods using pilot subcarriers in the estimation of CIR. The CIR estimation accuracy would be degraded in higher time-varying fading channels. In [13], we proposed the ICI mitigation method both for channel estimation and TDE methods in higher time-varying fading channels. The proposed method can achieve better BER performance. However, in demodulation processing, this method requires calculating the direct inverse at every OFDM symbol which leads to high computation complexity and not suitable in a practical system.

Although the proposed equalization methods [9][13] can achieve better BER performance than the conventional FDE method, however, all methods require the higher CIR estimation accuracy at every sampling time. This would cause higher computation complexity for calculation inverse matrix at every OFDM symbol.

The CIR estimation usually employs scattered pilot subcarriers which inserted into data subcarriers periodically in the frequency axis. The pilot subcarriers based CIR estimation can achieve higher CIR estimation accuracy only when the number of $\mathrm{N}$ point FFT/IFFT is equal to the number of $M$-point data subcarriers, which corresponds to the Nyquist sampling [14]. However, in the practical OFDM transmitter, a certain number of null subcarriers (zero paddings) are added at both ends of OFDM data subcarriers to reject the aliasing that occurs at the output of digital to analog (D/A) converter. Since actual CIR is occurred based on overall bandwidth including zero paddings, the estimated CIR by using the pilot subcarriers inserted within OFDM allocated bandwidth is different from actual CIR, especially when increasing the number of zero paddings. To solve this problem, we proposed a time-domain CIR estimation method by using a time-domain training sequence (TS), which can achieve higher CIR estimation accuracy even when the sampling rate of the OFDM signal is non-Nyquist sampling [13].

To reduce computation complexity and to solve the simultaneous equations for the equalization method in [9] and [13], an iterative method is employed instead of direct inverse matrix calculation. To employ an iterative method, the coefficients matrix of the simultaneous equations must be the square matrix. And to satisfy the above requirement, [9] employed the OFDM signal with guard interval
(GI) in which the coefficients matrix for the CIR over one data symbol period becomes the square matrix. On the other hand, the method in [13] employed the OFDM signal without GI in which the square coefficients matrix is generated by using the overlap and add (OLA) matrix operation after removing the interference from time-domain TS. The coefficients matrixes obtained from both [9] and [13] are entirely the same, which means that the time domain TS in [13] plays the role of GI in [9]. In [15], we proposed the TDE method with a partial differential solution for the OFDM signal without GI in quasi-static environments. The proposed TDE method shows much better BER performance than the conventional FDE method in which the data information is demodulated based on maximum likelihood (ML) estimation. From the results in [15], it can be expected that BER performance of proposed TDE without GI would have the possibility to improve BER performance further as compared with TDE methods in [9] and [13] even in higher time-varying fading channels.

To achieve superior BER performance both for quasi-static and higher time-varying fading channels, this paper firstly proposes the CIR estimation method by using the TS signal. The feature of the proposed CIR estimation method is to employ the TS signal added to each data symbol over one frame instead of conventional pilot subcarriers. Secondly, this paper proposes a low complexity equalization method for OFDM with the TS signal, which uses the CIR matrix in the time domain instead of using the CFR matrix in the conventional FDE method. The significant feature of the proposed TDE method is to employ the partial differential solution in solving the maximum likelihood (ML) equation of the expected timedomain information. As for the result of the minimization problem for solving ML equation, the coefficient matrix of the simultaneous equations in the proposed method becomes a square matrix, which can be solved iteratively without using inverse matrix calculation.

The rest of this paper is shown by the following. Section 2 introduces the problem of conventional FDE in higher time-varying fading channels. Section 3 describes the problem of Pilot subcarrier base CIR estimation in non-Nyquist sampling and also proposes a CIR estimation method by using the TS signal. Section 4 proposes the TDE method and lowcomplexity based TDE method in conjunction with estimated CIR at every sampling time. Section 5 presents different computer simulation results to verify the effectiveness of the proposed method as compared with conventional FDE methods. Section 6 draws some conclusions. 


\section{Problem of conventional FDE method in the higher time-varying fading channel}

\subsection{OFDM signal and channel models}

In OFDM systems, input data are encoded by a forward error correction (FEC) code [16]. The $M$ encoded data are modulated by quadrature amplitude modulation (QAM) method. The OFDM signal $X_{D}(m, k)$ at $k$-th subcarriers of an $m$-th symbol is mapped into a certain frequency band with $M$ subcarriers continuously from subcarrier number $N_{Z 1}$ to $N_{Z 2}\left(N_{Z 2}-N_{Z 1}+1=M\right)$ within $N$ subcarriers. The null subcarriers (zero paddings) are added at both ends of $M$ data subcarriers. The data information over $N$ subcarriers is given by,

$$
X_{Z}\left(m, k_{1}\right)= \begin{cases}0 \text { (Zero padding), } & 0 \leq k_{1} \leq N_{Z 1}-1 \\ X_{D}\left(m, k_{1}-N_{Z 1}\right), & N_{Z 1} \leq k_{1} \leq N_{Z 2} \\ 0 \text { (Zero padding), } & N_{Z 2}+1 \leq k_{1} \leq N\end{cases}
$$

where $(N-M)$ is the number of zero paddings $\left(N_{Z}\right)$ added at both ends of $M$ data subcarriers in the frequency domain. After added zero paddings, $X_{Z}\left(m, k_{1}\right)$ is converted into the time domain signal by mean of $N$-point inverse fast Fourier transform (IFFT) which can be given by,

$$
\begin{aligned}
x\left(m, n_{1}\right)= & \frac{1}{\sqrt{N}} \cdot \sum_{k_{1}=0}^{N-1} X_{Z}\left(m, k_{1}\right) \cdot e^{j \frac{2 \pi n_{1} k_{1}}{N}} \\
& , 0 \leq n_{1} \leq N-1
\end{aligned}
$$

where $x\left(m, n_{1}\right)$ is the transmitted OFDM signal in the time domain at an $n_{1}$-th sampling time of $m$-th symbol. For compensation of inter symbol interference (ISI), introduced by signal propagation through multipath fading channels, the OFDM signal employs the guard interval (GI) which is a copy of the last $N_{g}$ sampling time of data symbol inserted into the front of the signal [17] which can be expressed by,

$$
x_{G}\left(m, n_{2}\right)=\left\{\begin{array}{l}
x\left(m, n_{2}+N-N_{g}\right), \\
0 \leq n_{2} \leq N_{g}-1 \\
x\left(m, n_{2}-N_{g}\right), \\
N_{g} \leq n_{2} \leq N+N_{g}-1
\end{array}\right.
$$

In higher time-varying fading channels such as high-speed vehicles or trains communications [18], [19] the channel communication is often modeled as the Rician multipath fading channels. Here, the channel impulse response (CIR) $h_{l}\left(m, n_{2}\right)$ for $l$-th delay path at an $n_{2}$-th sampling time of $m$-th symbol in time-varying fading channels can be given by,

$$
h_{l}\left(m, n_{2}\right)=\sum_{l=0}^{L-1} h_{l}(m) \cdot \delta\left(n_{2}-\tau_{l}\right)
$$

where $h_{l}(m)$ is the complex amplitude of CIR for $l$-th delay path of an $m$-th symbol.

\subsection{Problem of conventional FDE method}

Assuming that the length of GI is longer than the maximum delay paths in the wireless channel and perfect synchronization timing is considered at the receiver. The received time-domain signal after removing GI at the $n_{2}$-th sample time during the $m$-th symbol can be expressed by,

$$
\begin{aligned}
r\left(m, n_{2}\right)= & \sum_{l=0}^{L-1} h_{l}\left(m, n_{2}\right) \otimes x_{G}\left(m, n_{2}\right)+w\left(m, n_{2}\right), \\
= & \sum_{l=0}^{L-1} h_{l}(m) \cdot x_{G}\left(m, n_{2}-N_{g}-l\right)+w\left(m, n_{2}\right), \\
& N_{g} \leq n_{2} \leq N+N_{g}-1
\end{aligned}
$$

where $\otimes$ represents the convolution. $r\left(m, n_{2}\right)$ is the received time-domain signal without GI and $w\left(m, n_{2}\right)$ is the zero-mean additive white Gaussian noise (AWGN) with variance $\sigma^{2}$. The $x_{G}\left(m, n_{2}-N_{g}-l\right)$ in Eq. (5) corresponds to $x\left(m, n_{1}-l\right)$, which given in Eq. (2) satisfies the followings,

$\begin{cases}n_{1}-l \geq 0, & x\left(m, n_{1}-l\right)=x\left(m, n_{1}-l\right) \\ n_{1}-l<0, & x\left(m, n_{1}-l\right)=x(m, N-l)\end{cases}$

The received time-domain signal $r\left(m, n_{2}\right)$ in Eq. (5) is converted to the frequency domain by $N$-point FFT which can be represented by,

$$
\begin{aligned}
R\left(m, k_{1}\right) & =\sum_{n_{2}=0}^{N-1} r\left(m, n_{2}\right) \cdot e^{-j \frac{2 \pi k_{1} n_{2}}{N}} \\
& =X_{Z}\left(m, k_{1}\right) \cdot H_{m}\left(k_{1}, k_{1}\right) \\
& +\underbrace{N-1}_{I C I} X_{Z}\left(m, k_{1}\right) \cdot H_{m}\left(k_{1}, k_{1}\right) \\
& +W\left(m, k_{1}\right), \quad 0 \leq k_{1} \leq N-1
\end{aligned}
$$

where $X_{Z}\left(m, k_{1}\right)$ is the transmitted data information, which given in Eq. (1) and $H_{m}$ is the channel frequency response (CFR). From Eq. (7), it can be seen that the second term is the ICI causes from timevarying fading channels. Fig. 1 shows a schematic 
diagram for the relationships between the CIR in the time domain and the channel frequency response (CFR) in the frequency domain. In the figure, the CFR is obtained by converting the CIR at a certain sampling time during one OFDM symbol period by using a discrete Fourier transform (DFT). Fig. 1.1 and 1.2 show the CIR and CFR in both quasi-static channels and high time-varying channels. From the figure, the CFR in high time-varying fading channels is changing according to the CIR at the different sampling times. From this fact, it is difficult to compensate for the time-varying fading distortion by using the conventional FDE method.

\section{CIR Estimation methods}

\subsection{Problem of conventional CIR estimation method}

In the conventional CIR estimation method, the pilot subcarrier usually employed, which be inserted periodically in frequency and time axis. The received frequency domain signal of an $m$-th symbol at $k_{1}$-th subcarrier in Eq. (7) can be rewritten by,

$$
R\left(m, k_{1}\right)=X_{Z}\left(m, k_{1}\right) \cdot H\left(m, k_{1}\right)+W\left(m, k_{1}\right)
$$

Here, the estimated CFR of the $m$-th symbol at $k_{p}$-th pilot subcarrier can be expressed by,

$$
\hat{H}\left(m, k_{p}\right)=\frac{R\left(m, k_{p}\right)}{P\left(m, k_{p}\right)}
$$

where $\hat{H}\left(m, k_{p}\right)$ is the estimated CFR, $R\left(m, k_{p}\right)$ received frequency-domain pilot subcarriers and $P\left(m, k_{p}\right)$ is the pilot pattern as known at the receiver. By using Eq. (9) with assumed that the $\hat{h}_{l}(m)$ is the unknown parameter, the estimated CFR is given by,

$$
\hat{H}\left(m, k_{p}\right)=\sum_{l=0}^{L-1} \hat{h}_{l}(m) \cdot e^{-j \frac{2 \pi k_{p}}{N}}
$$

By using Eqs. (9) and (10), the estimated CIR $\hat{h}_{l}(m)$ at every pilot symbol can be estimated by solving ML equation [17] under the constraint condition error between $\hat{H}_{l}\left(m, k_{p}\right)$ in Eqs. (9) and (10) is minimized as

$\Upsilon\left[h_{0} h_{1} \ldots h_{N_{g}-1}\right]=\arg \min \left[\sum_{p=0}^{N_{p}-1}\left|\hat{H}\left(m, k_{p}\right)-\sum_{l=0}^{N_{g}-1} \hat{h}_{l}(m) \cdot e^{-j \frac{2 \pi k k_{p}}{N}}\right|^{2}\right]$

where $N_{P}$ is the number of pilot subcarriers inserted
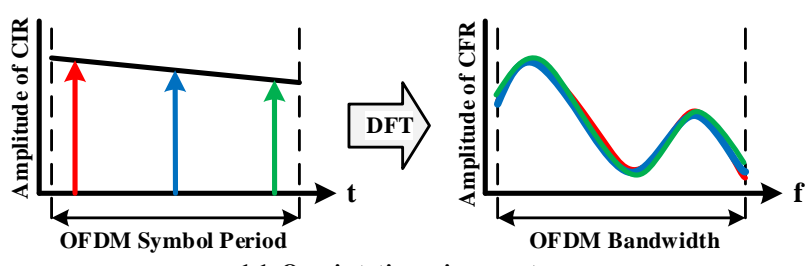

1.1 Quasi-static environments.

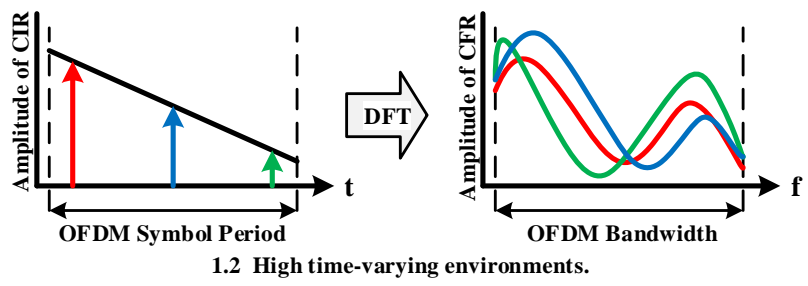

Figure. 1 Relationships between CIR and CFR

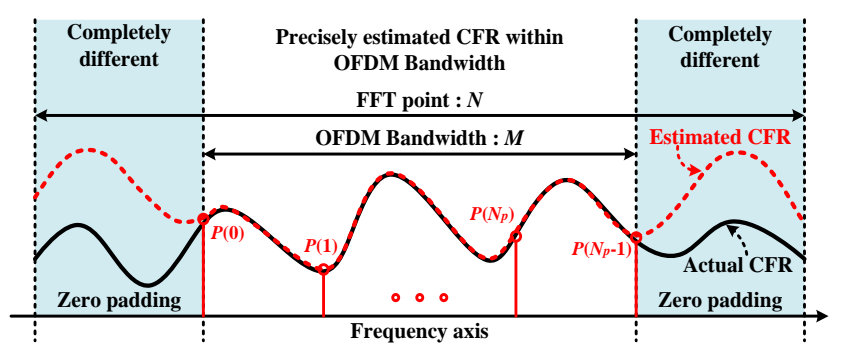

Figure. 2 Comparison of estimated CFR and actual CFR

in one OFDM symbol. Since the dependence from optimization parameters $\hat{h}_{l}(m)$ is linear in Eq. (11), the solution can be realized by the following MoorePenrose generalized matrix inversion.

$$
\underbrace{\left[\hat{h}_{l}(m)\right]}_{N_{g} \times 1}=\underbrace{\dagger\left[Z\left(k_{p}, l\right)\right]}_{N_{g} \times N_{p}} \cdot \underbrace{\left[\hat{H}\left(m, k_{p}\right)\right]}_{N_{p} \times 1}
$$

where $\dagger$ is the Moore-Penrose inverse matrix and $\left[\mathrm{Z}\left(k_{p}, l\right)\right]$ is given by,

$$
\left[Z\left(k_{p}, l\right)\right]=e^{-j \frac{2 \pi k_{p}}{N}}
$$

From Eqs. (10) to (13), there is a severe problem in the accuracy of CIR estimation from the received OFDM signal. Fig. 2 shows a comparison of the estimated CFR and actual CFR. The CFR can be obtained by performing a discrete Fourier transform (DFT) to CIR at a certain sampling time during one OFDM symbol period. In the Fig. 2, it can be observed that the estimated CFR by using pilot subcarriers can be estimated CFR precisely within OFDM bandwidth. However, the actual CFR in the real channel is entirely different from the estimated CFR. From this fact, the actual CIR is also completely different from the estimated CIR when the number of $N$-points FFT/IFFT is not equal to the 
number of $M$ data subcarriers, which corresponds to non-Nyquist sampling [13].

\subsection{Proposed CIR estimation method}

Fig. 3 shows the structure of the transmitter for the proposed method. At the transmitter side, the input data sequence is modulated. Then, added zero paddings and converted to the time domain signal $x\left(m, n_{1}\right)$ as same as the conventional OFDM signal. Fig. 4 shows the frame format employed in the proposed method. The time-domain TS1 and TS2 with the length of $N_{T S}$ are added at both ends of every data symbol and would be used in the estimation of CIR at every data symbol. The TS signal also used as the role of GI to avoid the ISI occurred in multipath fading channels. The transmitted time-domain signal $x_{T}\left(m, n_{2}\right)$ including both the TS1 and TS2 can be expressed by,

$$
\begin{aligned}
& x_{T}\left(m, n_{2}\right) \\
& = \begin{cases}d_{1}\left(m, n_{2}\right), & 0 \leq n_{2} \leq N_{T S}-1 \\
x\left(m, n_{2}-N_{T S}\right), & N_{T S} \leq n_{2} \leq N+N_{T S}-1 \\
d_{2}\left(m, n_{2}-N-N_{T S}\right), & N+N_{T S} \leq n_{2} \leq N+2 N_{T S}-1\end{cases}
\end{aligned}
$$

where $d_{1}(m, n)$ and $d_{2}(m, n)$ are the time domain TS1 and TS2 with the length of $N_{T S}$ samples $\left(0 \leq n \leq N_{T S}-\right.$ 1) of which data patterns are known at the receiver. For simplicity, this paper assumes the data patterns both for TS1 and TS2 are assumed to be the same as $d(m, n)$. The length of $N_{T S}$ should be taken longer than the length of delay paths $(L)$ as the same as the role of GI to avoid ISI. Here it is assumed that $h_{l}(m)$ is the constant during the period of TS1 even in higher time-varying fading channels. The actual received TS signal passed through the multi-path fading channels can be expressed by,

$$
\begin{aligned}
r_{T S}\left(m, n_{2}\right)= & \sum_{l=0}^{L-1} h_{l}(m) \cdot x_{T}\left(m, n_{2}-l\right)+w\left(m, n_{2}\right), \\
& 0 \leq n_{2} \leq N_{T S}-1
\end{aligned}
$$

where $h_{l}(m)$ is the ideal time-domain CIR for the $l$-th delay path at the $m$-th symbol. Assuming that the estimated CIR $\hat{h}_{l}(m)$ is unknown parameters, the expected received time-domain TS can be given by,

$$
\begin{gathered}
\hat{r}_{T S}\left(m, n_{2}\right)=\sum_{l=0}^{N_{T S}-1} \hat{h}_{l}(m) \cdot d\left(m, n_{2}-l\right), \\
0 \leq n_{2} \leq N_{T S}-1
\end{gathered}
$$

where $\hat{h}_{l}(m)$ is the estimated time-domain CIR for the

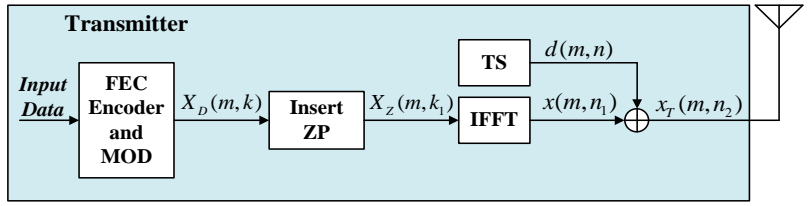

Figure. 3 Structure of the proposed TDE method

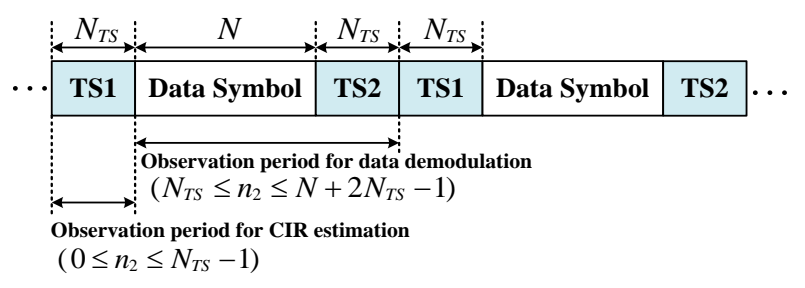

Figure. 4 Proposed frame format in the time domain

$l$-th delay path at the $m$-th symbol. The estimated CIR $\hat{h}_{l}(m)$ can be estimated by solving the following ML equation under constraint with minimizing the difference of actual received TS signal $r_{T S}\left(m, n_{2}\right)$ in Eq. (15) and expected received signal $\hat{r}_{T S}(m, n)$ in Eq. (16).

$$
\Upsilon=\underset{\hat{h}_{l}(m)}{\arg \min }\left[\sum_{n_{2}=0}^{N_{T S}-1}\left|r_{T S}\left(m, n_{2}\right)-\hat{r}_{T S}\left(m, n_{2}\right)\right|^{2}\right]
$$

The following equation can simply solve the ML equation in Eq. (17),

$$
r_{T S}\left(m, n_{2}\right)-\hat{r}_{T S}\left(m, n_{2}\right)=0, \quad 0 \leq n_{2} \leq N_{T S}-1
$$

In the result of Eq. (18), by minimizing the difference of actual received TS signal and expected received TS signal can be expressed by,

$$
r_{T S}\left(m, n_{2}\right)=\sum_{l=0}^{N_{T_{s}}-1} \hat{h}_{l}(m) \cdot d\left(m, n_{2}-l\right), 0 \leq n_{2} \leq N_{T S}-1
$$

Finally, Eq. (19) can be solved as the results on the following simultaneous equations.

$$
\underbrace{\left[\hat{h}_{l}(m)\right]}_{N_{T S} \times 1}=\underbrace{\left[d\left(m, n_{2}-l\right)\right]^{-1}}_{N_{T S} \times N_{T S}} \cdot[\underbrace{\left[r_{T S}\left(m, n_{2}\right)\right]}_{N_{T S} \times 1}
$$

where $[\cdot]^{-1}$ is matrix inversion operator and $d\left(m, n_{2}-l\right)$ given in Eq. (20) satisfies the followings,

$$
\left\{\begin{array}{l}
n_{2}-l \geq 0, \quad d\left(m, n_{2}-l\right)=d\left(m, n_{2}-l\right) \\
n_{2}-l<0, \quad d\left(m, n_{2}-l\right)=d\left(m, N_{T S}-l\right)
\end{array}\right.
$$

where $d(m, n)$ is the time domain TS and its data pattern is known at the receiver. The inverse matrix $\left[d\left(m, n_{2}-l\right)\right]^{-1}$ in Eq. (20) can be calculated in advance and leads to the considerable reduction of computation complexity in the estimation of CIR at every symbol. By using the estimated $\left[\hat{h}_{l}(m)\right]$ at every 
symbol, the estimated CIR at every sampling time $\left[\hat{h}_{l}\left(m, n_{2}\right)\right]$ can be estimated by using the cubic spline interpolation method [20] between two consecutive estimated CIRs of $\left[\hat{h}_{l}(m)\right]$ and $\left[\hat{h}_{l}(m+1)\right]$.

Fig. 5 shows a schematic diagram for the cubic spline interpolation method to estimate the CIR at every sampling time. The estimation accuracy of CIR at every sampling time for the proposed method will be evaluated in section 5 as compared with the conventional pilot base CIR estimation when the sampling rate of the OFDM signal is a non-Nyquist sampling.

\section{Time domain equalization methods}

\subsection{TDE with OLA method}

The proposed equalization methods in [9] and [13] show almost the same BER performance when using the ideal CIR $h_{l}\left(m, n_{2}\right)$ at every sampling time for data demodulation. However, [9] considers the estimated CIR $\hat{h}_{l}\left(m, n_{2}\right)$ at every sampling time of using the pilot subcarriers based CIR estimation method. The estimation accuracy of CIR at every sampling time is no more satisfied as described in section 3. On the other hand, in [13] using the time domain TS for estimation of CIR at every sampling time, which can be estimated CIR very precisely even when the sampling rate of OFDM signal is the nonNyquist sampling and also even in higher timevarying fading channels [13].

Fig. 6 shows the structure of the receiver for the TDE with the OLA method. By assuming the ideal CIR $h_{l}\left(m, n_{2}\right)$ at every sampling time, the received data signal $r_{D}\left(m, n_{2}\right)$ at every sampling time during the observation period for data demodulation from $N_{T S}$ to $N+2 N_{T S}-1$ can be expressed by,

$$
\begin{aligned}
r_{D}\left(m, n_{2}\right)= & \sum_{l=0}^{L-1} h_{l}\left(m, n_{2}\right) \cdot x_{T}\left(m, n_{2}-l\right)+w\left(m, n_{2}\right), \\
& N_{T S} \leq n_{2} \leq N+2 N_{T S}-1
\end{aligned}
$$

where $x_{T}\left(m, n_{2}-l\right)$ given in (14) satisfies the followings,

$$
\begin{cases}n_{2}-l \leq N_{T S}-1, & x_{T}\left(m, n_{2}-l\right)=d\left(m, n_{2}-l\right) \\ n_{2}-l \geq N+N_{T S}, & x_{T}\left(m, n_{2}-l\right)=d\left(m, n_{2}-N-N_{T S}-l\right)\end{cases}
$$

The actual received data signal in Eq. (22) includes the interferences of TS, which are added at the start and the end of data symbol from the TS1 and TS2, respectively. By using the estimated $\mathrm{CIR} \hat{h}_{l}\left(m, n_{2}\right)$ at every sampling time and the data pattern of $d\left(m, n_{2}\right)$ both for TS1 and TS2 which is known at the receiver,

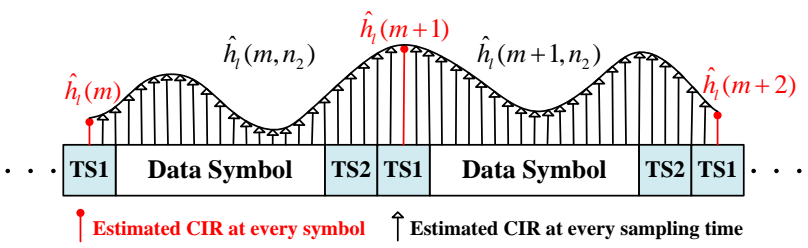

Figure. 5 Estimation of CIR at every sampling time by using a cubic spline interpolation method

the interference of TS added at both ends of the timedomain data signal can be removed by the following equation.

$$
r_{F}\left(m, n_{2}\right)=\left\{\begin{aligned}
r_{D}\left(m, n_{2}\right)- & \sum_{l=n_{2}-N_{T S}+1}^{N_{T S}-1} \hat{h}_{l}\left(m, n_{2}\right) \cdot d\left(m, n_{2}-l\right), \\
& \left(N_{T S} \leq n_{2} \leq 2 N_{T S}-2\right) \\
r_{D}\left(m, n_{2}\right), & \left(2 N_{T S}-1 \leq n_{2} \leq N+N_{T S}-1\right) \\
r_{D}\left(m, n_{2}\right)- & \sum_{l=0}^{n_{2}-N-N_{T S}} \hat{h}_{l}\left(m, n_{2}\right) \cdot d\left(m, n_{2}-N-N_{T S}-l\right), \\
& \left(N+N_{T S} \leq n_{2} \leq N+2 N_{T S}-2\right)
\end{aligned}\right.
$$

where $r_{F}\left(m, n_{2}\right)$ is the received time-domain signal after removing the interference of TS from the actually received signal $r_{D}\left(m, n_{2}\right)$ in Eq. (22). When the transmitted time-domain data $x\left(m, n_{1}\right)$ given in Eq. (2) is assumed as the unknown parameters, the expected time domain received data $\hat{r}_{E}\left(m, n_{2}\right)$ without the interference of TS which corresponds to Eq. (24) can be given by,

$$
\hat{r}_{E}\left(m, n_{2}\right)=\left\{\begin{array}{c}
\sum_{l=0}^{n_{2}-N_{T S}} \hat{h}_{l}\left(m, n_{2}\right) \cdot \hat{x}\left(m, n_{2}-N_{T S}-l\right), \\
\left(N_{T S} \leq n_{2} \leq 2 N_{T S}-2\right) \\
\sum_{l=0}^{N_{T S}-1} \hat{h}_{l}\left(m, n_{2}\right) \cdot \hat{x}\left(m, n_{2}-N_{T S}-l\right), \\
\left(2 N_{T S}-1 \leq n_{2} \leq N+N_{T S}-1\right) \\
\sum_{l=n_{2}-N-N_{T S}+1}^{N_{T S}-1} \hat{h}_{l}\left(m, n_{2}\right) \cdot \hat{x}\left(m, n_{2}-N_{T S}-l\right), \\
\left(N+N_{T S} \leq n_{2} \leq N+2 N_{T S}-2\right)
\end{array}\right.
$$

The maximum likelihood (ML) can estimate the unknown parameter of time-domain data $\hat{x}\left(m, n_{1}\right)$, that ML equation solve under the constraint with minimizing the difference between the actually received data $r_{F}\left(m, n_{2}\right)$ in Eq. (24) and the expected received data $\hat{r}_{E}\left(m, n_{2}\right)$ in Eq. (25).

$$
\Upsilon=\underset{\hat{x}\left(m, n_{1}\right)}{\arg \min }\left[\sum_{n_{2}=N_{T S}}^{N+2 N_{T S}-2}\left|r_{F}\left(m, n_{2}\right)-\hat{r}_{E}\left(m, n_{2}\right)\right|^{2}\right]
$$

The following equations can solve the ML equation in Eq. (26). 


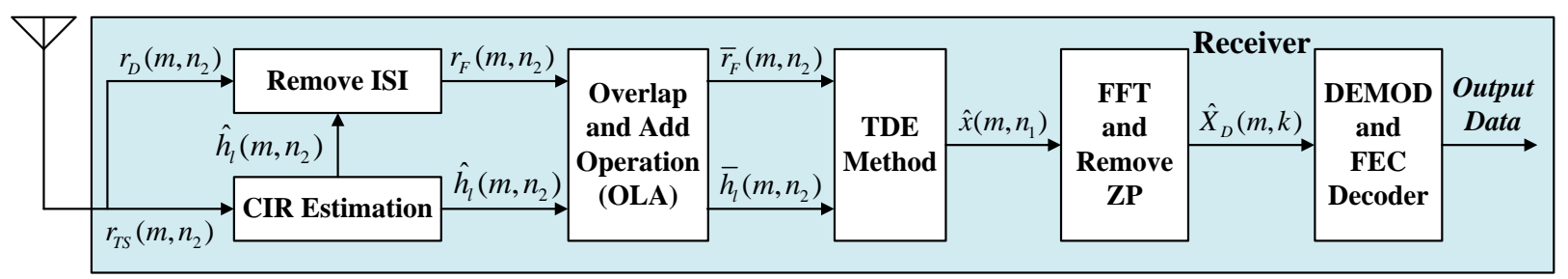

Figure. 6 Receiver structure for TDE with the OLA method

$r_{F}\left(m, n_{2}\right)-\hat{r}_{E}\left(m, n_{2}\right)=0, \quad N_{T S} \leq n_{2} \leq N+N_{T S}-1$

By using the condition in Eq. (27) and substituting Eq. (25) into Eq. (27), Eq. (27) can be expressed by the following simultaneous equations.

$$
r_{F}\left(m, n_{2}\right)=\left\{\begin{array}{c}
\sum_{l=0}^{n_{2}-N_{T S}} \hat{h}_{l}\left(m, n_{2}\right) \cdot \hat{x}\left(m, n_{2}-N_{T S}-l\right), \\
N_{T S} \leq n_{2} \leq 2 N_{T S}-2 \\
\sum_{l=0}^{N_{T S}-1} \hat{h}_{l}\left(m, n_{2}\right) \cdot \hat{x}\left(m, n_{2}-N_{T S}-l\right), \\
2 N_{T S}-1 \leq n_{2} \leq N+N_{T S}-1 \\
\sum_{l=n_{2}-N-N_{T S}+1}^{N_{T S}-1} \hat{h}_{l}\left(m, n_{2}\right) \cdot \hat{x}\left(m, n_{2}-N_{T S}-l\right), \\
N+N_{T S} \leq n_{2} \leq N+2 N_{T S}-2
\end{array}\right.
$$

where $\hat{x}\left(m, n_{2}-N_{T S}-l\right)$ in Eq. (28) correspond to $\hat{x}\left(m, n_{1}-l\right)$ with condition $\left(0 \leq n_{1} \leq N-1\right)$. Finally, Eq. (28) is resulted in the following matrix operation.

$$
\underbrace{\left[r_{F}\left(m, n_{2}\right)\right]}_{\left(N+N_{T s}-1\right) \times 1}=\underbrace{\left[\hat{h}_{l}\left(m, n_{2}\right)\right]}_{\left(N+N_{T s}-1\right) \times N} \cdot \underbrace{\left[\hat{x}\left(m, n_{1}\right)\right]}_{N \times 1}
$$

where $\left[r_{F}\left(m, n_{2}\right)\right]$ received a time-domain signal with a length of $\left(N+N_{T s}-1\right) \times 1$ matrix. $\left[\hat{h}_{l}\left(m, n_{2}\right)\right]$ is timedomain CIR with a length of $\left(N+N_{T S}-1\right) \times N$ matrix. Here $\left[r_{F}\left(m, n_{2}\right)\right]$ in Eq. (29) can be spilled into its upper $N \times 1$ part and it is lower $\left(N_{T S}-1\right) \times 1$ part. Similarly, $\left[\hat{h}_{l}\left(m, n_{2}\right)\right]$ can be split into its upper $N \times N$ part and its lower $\left(N_{T S}-1\right) \times N$ part. By adding the lower elements to upper elements, $\left[r_{F}\left(m, n_{2}\right)\right]$ and $\left[\hat{h}_{l}\left(m, n_{2}\right)\right]$ can be given by the following matrixes with the size of $N \times 1$ and $N \times N$, respectively.

$$
\begin{array}{r}
{\left[\bar{r}_{F}\left(m, n_{2}\right)\right]=\Phi\left[r_{F}\left(m, n_{2}\right)\right]=\left[I_{N} \vdots \frac{I_{N_{T S}-1}}{0}\right] \cdot\left[r_{F}\left(m, n_{2}\right)\right] \quad(30)} \\
{\left[\bar{h}_{l}\left(m, n_{2}\right)\right]=\Phi\left[\hat{h}_{l}\left(m, n_{2}\right)\right]=\left[I_{N} \vdots \frac{I_{N_{T S}-1}}{0}\right] \cdot\left[\hat{h}_{l}\left(m, n_{2}\right)\right]}
\end{array}
$$

where $\Phi$ denotes an overlap-and-add (OLA) operator which makes circular convolution feasible. From Eqs. (30) and (31), Eq. (29) becomes the following matrix operations with the size of the $N \times N$ matrix.

$$
\underbrace{\left[\bar{r}_{F}\left(m, n_{2}\right)\right]}_{N \times 1}=\underbrace{\left[\bar{h}_{l}\left(m, n_{2}\right)\right]}_{N \times N} \cdot \underbrace{\left[\hat{x}\left(m, n_{1}\right)\right]}_{N \times 1}
$$

From Eq. (32), it can be observed that the CIR matrix becomes the circular matrix, which is the same as the OFDM signal with GI when assuming the quasi-static multipath fading channels [13]. The expected timedomain signal can be estimated by using the direct calculation of the inverse matrix which is given by,

$$
\underbrace{\left[\hat{x}\left(m, n_{1}\right)\right]}_{N \times 1}=[\underbrace{\left[\bar{h}_{l}\left(m, n_{2}\right)\right]}_{N \times N}]^{-1} \cdot \underbrace{\left[\bar{r}_{F}\left(m, n_{2}\right)\right]}_{N \times 1}
$$

where $\left[\bar{h}_{l}\left(m, n_{2}\right)\right]^{-1}$ is the inverse of the channel matrix and $\left[\hat{x}\left(m, n_{1}\right)\right]$ is the estimated time-domain data information matrix. Since the TDE with the OLA method can track the time-varying CIR at every sampling time as given in Eq. (28), it is possible to mitigate the ICI and expected to achieve better BER performance than the conventional FDE method in higher time-varying fading channels. In Eq. (33), the estimated time-domain $\hat{x}\left(m, n_{1}\right)$ can be solved simply by using the inverse matrix calculation with the size of $N \times N$. The computational complexity of the TDE with the OLA method can be considered as matrix inversion is $O\left(N^{3}\right)$, and matrix multiplication is $O\left(N^{2}\right)$. Here, the total computational complexity for the TDE with the OLA method is to order $N^{3}+N^{2}$ at every data symbol.

\subsection{Proposed TDE with partial differential method}

In the TDE with the OLA method, the BER performance would be degraded from OLA operator in (30) and (31) due to the summation of unexpected phase noise from AWGN and multipath channel due to its upper $N \times 1$ and lower $\left(N_{T S}-1\right) \times 1$ parts. Fig. 7 shows the structure of the receiver for the proposed TDE with the partial differential method. The 


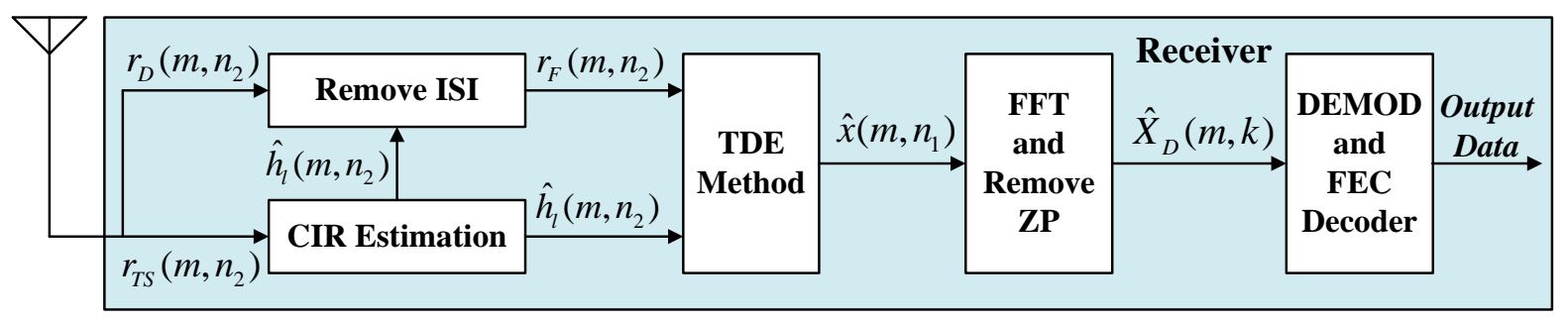

Figure. 7 Receiver structure for proposed TDE with the partial differential method

maximum likelihood (ML) can estimate the unknown parameter of time-domain data $\hat{x}\left(m, n_{1}\right)$ in Eq. (2), that ML equation solve under the constraint with minimizing the difference between the actually received data $r_{F}\left(m, n_{2}\right)$ in Eq. (24) and the expected received data $\hat{r}_{E}\left(m, n_{2}\right)$ in Eq. $(25)$.

$$
\Upsilon=\underset{\hat{x}(m, s)}{\arg \min }\left[\sum_{n_{2}=N_{T S}}^{N+2 N_{T S}-2}\left|r_{F}\left(m, n_{2}\right)-\hat{r}_{E}\left(m, n_{2}\right)\right|^{2}\right]
$$

The ML equation (34) can be solved by taking the partial differentiation for all unknown parameters of $\hat{x}^{*}(m, s)$ which can be expressed by,

$$
\frac{\partial \Upsilon}{\partial \hat{x}^{*}(m, s)}=\frac{\partial\left(\sum_{n_{2}=N_{T S}}^{N+2 N_{T S}-2}\left|r_{F}\left(m, n_{2}\right)-\hat{r}_{E}\left(m, n_{2}\right)\right|^{2}\right)}{\partial \hat{x}^{*}(m, s)}=0,
$$

where $*$ represents the conjugate complex number. By using Eq. (35), the ML equation (34) can be expressed by the following simultaneous equations with $N$ unknown parameters of $\hat{x}\left(m, n_{1}\right)$.

$$
\underbrace{[b(m, s)]}_{N \times 1}=[\underbrace{\left[A_{m}\left(s, n_{1}\right)\right]}_{N \times N} \cdot \underbrace{\left[\hat{x}\left(m, n_{1}\right)\right]}_{N \times 1}
$$

where $b(m, s)$ and $A_{m}\left(s, n_{1}\right)$ can be expressed by,

$$
\begin{aligned}
& b(m, s)=\sum_{n_{2}=N_{T S}}^{N+2 N_{T S}-2} r_{F}\left(m, n_{2}\right) \frac{\partial \hat{r}_{E}^{*}\left(m, n_{2}\right)}{\partial \hat{x}^{*}(m, s)}, 0 \leq s \leq N-1 \\
& A_{m}\left(s, n_{1}\right)=\sum_{n_{2}=N_{T S}}^{N+2 N_{T S}-2} \hat{r}_{E}\left(m, n_{2}\right) \frac{\partial \hat{r}_{E}^{*}\left(m, n_{2}\right)}{\partial \hat{x}^{*}(m, s)}, 0 \leq n_{1} \leq N-1
\end{aligned}
$$

where,

$$
\begin{aligned}
s & =n_{2}-N_{T S}-l \text { in } \partial \hat{r}_{E}^{*}\left(m, n_{2}\right) / \partial \hat{x}^{*}(m, s) \\
n_{1} & =n_{2}-N_{T S}-l \text { in } \hat{r}_{E}\left(m, n_{2}\right)
\end{aligned}
$$

From Eq. (36), the unknown parameters $\hat{x}\left(m, n_{1}\right)$ can be solved by using the inverse matrix of $\left[A_{m}\left(s, n_{1}\right)\right]$ which is given by,

$$
\underbrace{\left[\hat{x}\left(m, n_{1}\right)\right]}_{N \times 1}=[\underbrace{\left.A_{m}\left(s, n_{1}\right)\right]^{-1}}_{N \times N} \cdot \underbrace{[b(m, s)]}_{N \times 1}
$$

Similar to the TDE with OLA method, the estimated time-domain signal $\hat{x}\left(m, n_{1}\right)$ in Eq. (41), the $M$ encoded data information $\hat{X}_{D}(m, k)$ can be demodulated by processing $N$-points FFT, remove zero paddings and demodulation which are all the opposite processing at the transmitter side. Here, the total computational complexity for proposed TDE with the partial differential method is order $N^{3}+N^{2}$ at every OFDM symbol. From Eqs. (33) and (41), The computational complexity for both proposed TDE with OLA and proposed TDE with partial differential methods is significantly high and is not suitable for practical implementation because it is required to calculate the inverse matrix at every data symbol.

\subsection{Proposed TDE with iterative method}

The coefficients matrix $\left[A_{m}\left(s, n_{1}\right)\right]$ after taking the partial differentiation in Eq. (36) which is the square matrix with a size of $N \times N$. This means that matrix $\left[A_{m}\left(s, n_{1}\right)\right]$ can be solved by using an iterative calculation. The conjugate gradient squared (CGS) algorithm [21] is well known as one of the iterative methods which can solve a system of $N$ linear equations with $N$ unknown parameters. Let us consider the system $A \hat{x}=b$, where $A$ corresponds to $A_{m}\left(s, n_{1}\right)$ in Eq. (36) and it is the Hermitian and positive definite matrix with the size of $N \times N$. The exact CGS solution can be obtained after at most $N$ steps. Hence, stopping the iteration after $N_{\text {iter }}(<N)$ steps would yield an approximate solution. In the demodulation of every data symbol, the CGS algorithm minimizes iteratively calculates the cost function in a reduced-rank Krylov subspace. When the spectral condition number of the matrix $A$ is too high, a preconditioned matrix $D$ is employed which is called the precondition CGS (PCGS) algorithm. The PCGS algorithm solves the system by,

$$
D^{-1} A \hat{x}=D^{-1} b
$$


Table 1. Preconditioned conjugate gradient squared (PCGS) algorithm

\begin{tabular}{|c|c|}
\hline \multicolumn{2}{|l|}{ PCGS Algorithm } \\
\hline \multicolumn{2}{|l|}{ Initialization : } \\
\hline \multicolumn{2}{|l|}{$\begin{array}{l}\hat{x}_{0} \text { is an initial guess; } \\
r_{0}=b-A \hat{x}_{0}\end{array}$} \\
\hline$\tilde{r_{0}}=r_{0} ; \rho_{0}=\left(r_{0}, \tilde{r_{0}}\right)$ & $O(N)$ \\
\hline$\beta_{-1}=\rho_{0} ; p_{-1}=q_{0}=0$ & $O(N)$ \\
\hline 1. for $i=0,1,2, \ldots, N_{\max }$ do & \\
\hline 2 & $O(N)$ \\
\hline$p_{i}=u_{i}+\beta_{i-1}\left(q_{i}+\beta_{i-1} p_{i-1}\right)$ & $O(N)$ \\
\hline$\hat{p}=D^{-1} p_{i}$ & $O(N)$ \\
\hline$\hat{v}=A \hat{p_{0}}$ & $O\left(N^{2}\right)$ \\
\hline$\alpha_{i}=\rho_{i} /\left(\tilde{r_{0}}, \hat{v}\right)$ & $O(N)$ \\
\hline$q_{0}=u_{i}-\alpha_{i} \hat{v}$ & $O(N)$ \\
\hline$\hat{u}=D^{-1}\left(u_{i}+q_{i+1}\right)$ & $O(N)$ \\
\hline$\hat{x}_{i+1}=\hat{x}_{i}+\alpha_{i} \hat{u}$ & $O(N)$ \\
\hline $\begin{array}{l}\text { 10. if } N M S E \text { in }(44)>\text { Threshold } \\
\text { level }(T O L)\end{array}$ & $O(N)$ \\
\hline 11. return; else & \\
\hline$r_{i+1}=r_{i}-\alpha_{i} A \hat{u}$ & $O\left(N^{2}\right)$ \\
\hline 13. $\rho_{i+1}=\left(r_{0}, \tilde{r}_{i+1}\right)$ & $O(N)$ \\
\hline $\begin{array}{l}\text { 14. } \quad \alpha_{i}=\rho_{i+1} / \rho_{i} \text {; } \\
\text { 15. end for }\end{array}$ & $O(N)$ \\
\hline
\end{tabular}

where the inversion of matrix $D$ should be a computationally efficient operation. For the rest of our analysis, we assume for simplicity that matrix $D(m)$ is the diagonal matrix of $A_{m}\left(s, n_{1}\right)$ at $m$-th symbol. The initial solution of $\hat{x}\left(m, n_{1}\right)$ is given by,

$$
\underbrace{\left[\hat{x}\left(m, n_{1}\right)\right]}_{N \times 1}]^{(0)}=\underbrace{[D(m)]^{-1}}_{N \times N} \cdot \underbrace{[b(m, s)]}_{N \times 1}
$$

The reduced-rank PCGS method is described analytically in Table 1 . The repetition of the PCGS algorithm is stopped when the following normalized mean square error (NMSE) between the $i$-th and $(i+1)$ th solutions of $\left[\hat{x}\left(m, n_{1}\right)\right]$ is smaller than the predetermined threshold level (TOL).

$$
N M S E=\frac{\sum_{n_{1}=0}^{N-1}\left|\left[\hat{x}\left(m, n_{1}\right)\right]^{(i)}-\left[\hat{x}\left(m, n_{1}\right)\right]^{(i+1)}\right|^{2}}{\sum_{n_{1}=0}^{N-1}\left|\left[\hat{x}\left(m, n_{1}\right)\right]^{(i)}\right|^{2}}
$$

In the proposed iterative based TDE with the PCGS algorithm, the following procedures are repeated up to either the number of iterations reaches to the predetermined maximum number $\left(N_{\max }\right)$ or $N M S E$ values less than the predetermined threshold level $(T O L)$.

Step1: The maximum iteration number is set to $N_{\max }$

$\left(N_{\max }=5\right)$, the threshold level is set to $T O L$, and the initial solution of $\left[\hat{x}\left(m, n_{1}\right)\right]^{(0)}$ is given by Eq. (43).
Step2: Calculate the $i$-th solution of $\left[\hat{x}\left(m, n_{1}\right)\right]^{(i)}$ by the PCGS algorithm and calculate the NMSE by Eq. (44).

Step3: Compare the NMSE obtained at the i-th iteration with the predetermined threshold level of TOL. If the NMSE is less than TOL, the $\left[\hat{x}\left(m, n_{1}\right)\right]^{(i)}$ is output as the estimated data information. If not, repeat the same procedures. If the number of iterations reaches to predetermined $N_{\max }$, $\left[\hat{x}\left(m, n_{1}\right)\right]^{\left(N_{\max }\right)}$ is output as the estimated data information.

From Table 1, it is clear that the computational complexity is determined by the initialization of line 5 and line 12. Specifically, the total computational complexity order for the demodulation of each OFDM symbol can be expressed by $N_{\text {iter }}\left(2 N^{2}+10 N\right)+N^{2}+2 N$. The following equation defines the ratio of computation complexity between TDE with the inverse matrix and TDE with an iterative method for the whole OFDM frame.

$$
\begin{aligned}
R & =\frac{N_{S} \cdot\left\{N_{\text {Aver }} \cdot\left(2 N^{2}+10 N\right)+N^{2}+2 N\right\}}{N_{S} \cdot\left\{N^{3}+N^{2}\right\}} \\
& =\frac{N_{\text {Aver }} \cdot\left(2 N^{2}+10 N\right)+N^{2}+2 N}{N^{3}+N^{2}}
\end{aligned}
$$

where $N_{S}$ is the number of data symbols in one OFDM frame, and $N_{\text {Aver }}$ is the average number of iterations in one OFDM frame.

\section{Performance evaluation}

This section presents various simulation results to verify the effectiveness of proposed TDE with the iterative method as compared with the conventional FDE method [14] and the TDE with the OLA method [13]. Table 2 shows the list of simulation parameters, which use the following evaluations. The conventional FDE method employs pilot subcarriers with an interval of pilot subcarriers in the frequency axis is FIP=4, and the pilot symbol includes the data subcarriers, which added at every time axis $(\mathrm{TIP}=1)$.

The proposed CIR estimation method is employed both of the proposed TDE with iterative and TDE with OLA methods. The communication channel is modeled by the Rician multipath fading, which usually experienced by the user on the higher moving vehicle [18]. We assume the normalized Doppler frequency $R_{D}=f_{d \max } / \Delta f(\%)$, which is defined by the ratio of maximum Doppler frequency $f_{d \max }$ to the subcarrier spacing $\Delta f$ is employed as the measure of mobile condition.

Fig. 8 shows the time domain CIR estimation accuracy at every sampling time, which evaluated by the normalized mean square error (NMSE) for the 
pilot subcarriers and the proposed TS methods when changing the number of zero paddings both sides of data subcarriers $\left(N_{Z}\right)$. From the simulation results in Fig. 8, it shows that the proposed TS method can keep the higher estimation accuracy regardless of the number of $N_{Z}$. While the estimation accuracy for the pilot subcarriers method is getting worse when increasing the number of $N_{Z}$ and $R_{D}$, respectively.

Fig. 9 shows BER performance for proposed TDE with iterative method when changing the threshold level (TOL). In the simulation, normalized Doppler frequency $R_{D}$ is $15 \%$, which corresponds to the vehicle speed of $286 \mathrm{~km} / \mathrm{hrs}$. In Fig. 9, it can be observed that the BER performance of proposed TDE with the iterative method is very close to that for the inverse matrix calculation method when the threshold level TOL is less than 0.01 with regardless of operation $\mathrm{C} / \mathrm{N}$. From the results in Fig. 9, the threshold level TOL for the NMSE is taken by $T O L=0.01$ in the next following evaluations.

Table 2. Simulation parameters

\begin{tabular}{|c|c|c|}
\hline \multicolumn{2}{|c|}{ Parameter } & Value \\
\hline \multicolumn{2}{|c|}{ Number of FFT points $(N)$} & 128 \\
\hline \multicolumn{2}{|c|}{ Number of subcarriers $(M)$} & 96 \\
\hline \multicolumn{2}{|c|}{ Number of zero paddings $\left(N_{Z}\right)$} & 32 \\
\hline \multirow{2}{*}{$\begin{array}{l}\text { Convolutional } \\
\text { FEC }\end{array}$} & Rate & $1 / 2$ \\
\hline & Constraint length & 7 \\
\hline \multirow{4}{*}{$\begin{array}{l}\text { Conventional } \\
\text { FDE [14] }\end{array}$} & Modulation for pilot & QPSK \\
\hline & $\begin{array}{l}\text { Pilot interval } \\
(\mathrm{FIP}, \mathrm{TIP})\end{array}$ & $(4,1)$ \\
\hline & $\begin{array}{l}\text { Length of the guard } \\
\text { interval (GI) }\end{array}$ & 16 \\
\hline & Symbol duration $\left(T_{S}\right)$ & $108 \mu \mathrm{s}$ \\
\hline \multirow{3}{*}{$\begin{array}{l}\text { Proposed } \\
\text { TDE }\end{array}$} & Modulation for TS & 16QAM \\
\hline & $\begin{array}{l}\text { Length of training } \\
\text { sequence (TS) }\end{array}$ & 16 \\
\hline & Symbol duration $\left(T_{S}\right)$ & $120 \mu \mathrm{s}$ \\
\hline \multicolumn{2}{|c|}{ Modulation for data subcarrier } & 16QAM \\
\hline \multicolumn{2}{|c|}{ Number of symbols per one frame $\left(N_{S}\right)$} & 33 \\
\hline \multicolumn{2}{|c|}{ Allocated bandwidth } & $1 \mathrm{MHz}$ \\
\hline \multicolumn{2}{|c|}{ Radio frequency } & $5.9 \mathrm{GHz}$ \\
\hline \multicolumn{3}{|c|}{ Rician multipath fading channel model } \\
\hline \multicolumn{2}{|c|}{ Rician factor $(K)$} & $6 \mathrm{~dB}$ \\
\hline \multicolumn{2}{|c|}{ Delay profile } & Exponential \\
\hline \multicolumn{2}{|c|}{ Decay constant } & $-1 \mathrm{~dB}$ \\
\hline \multicolumn{2}{|c|}{ Number of delay paths $(L)$} & 14 \\
\hline \multicolumn{2}{|c|}{ Number of scattered rays } & 20 \\
\hline
\end{tabular}

Table 3. Comparison of complexity ratios

\begin{tabular}{|c|c|c|c|c|c|c|}
\hline \multirow{3}{*}{$\mathrm{C} / \mathrm{N}$} & \multicolumn{6}{|c|}{$\begin{array}{l}\text { Proposed iterative based TDE method }(N=128, \\
\text { TOL }=0.01)\end{array}$} \\
\hline & \multicolumn{2}{|c|}{$f_{d \max } / \Delta f=10 \%$} & \multicolumn{2}{|c|}{$f_{d \max } / \Delta f=15 \%$} & \multicolumn{2}{|c|}{$f_{d \max } / \Delta f=20 \%$} \\
\hline & $N_{\text {Aver }}$ & $R$ & $N_{\text {Aver }}$ & $R$ & $N_{\text {Aver }}$ & $R$ \\
\hline $14 \mathrm{~dB}$ & 4.25 & 0.076 & 4.29 & 0.076 & 4.37 & 0.078 \\
\hline $17 \mathrm{~dB}$ & 3.95 & 0.071 & 4.01 & 0.072 & 4.08 & 0.073 \\
\hline $20 \mathrm{~dB}$ & 3.81 & 0.069 & 3.86 & 0.070 & 3.94 & 0.071 \\
\hline
\end{tabular}

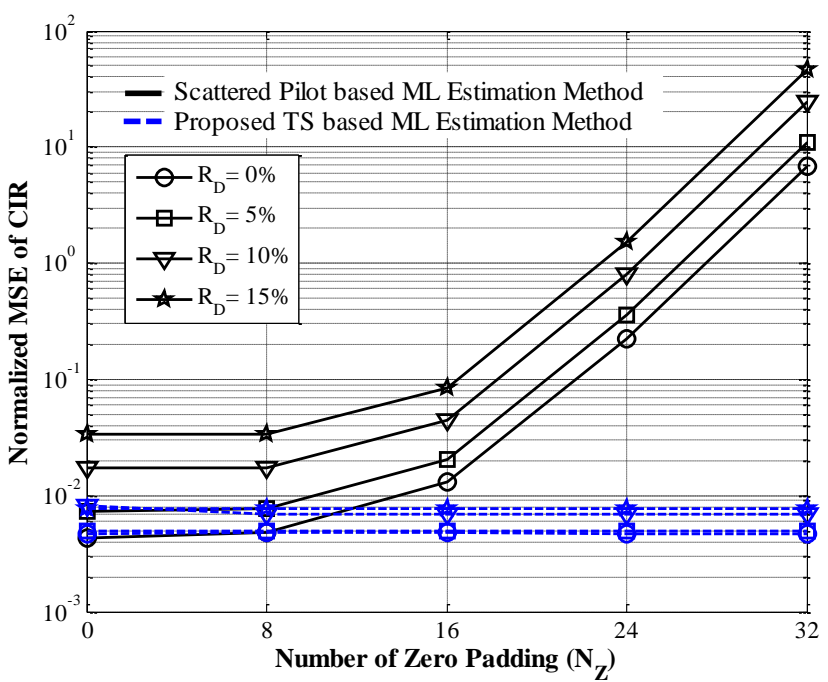

Figure. 8 CIR estimation accuracy (NMSE) for the proposed method at $\mathrm{C} / \mathrm{N}=20 \mathrm{~dB}$

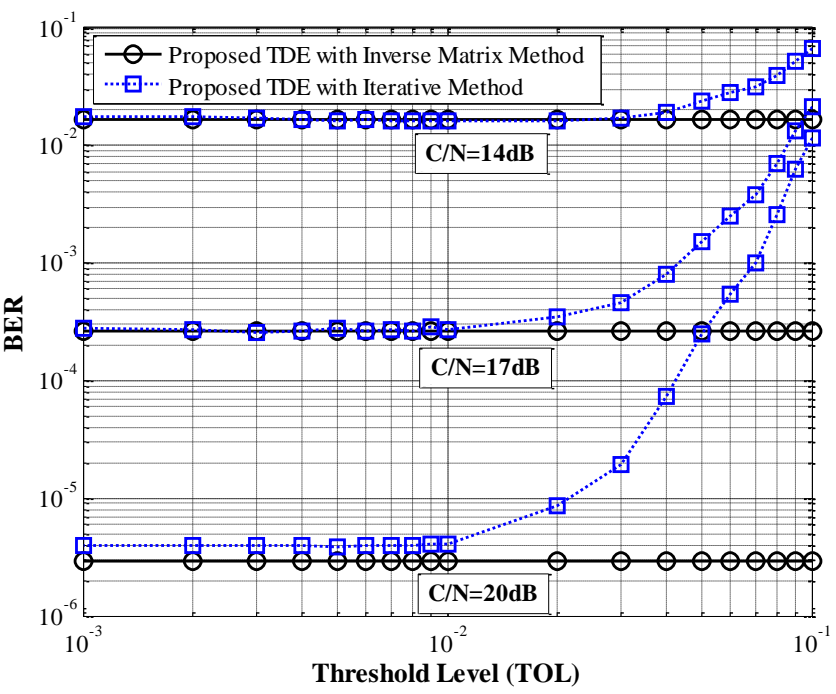

Figure. 9 BER performance for the proposed method when changing TOL at $R_{D}=15 \%$

Fig. 10 shows the average number of iterations for the proposed TDE with iterative method when changing $R_{D}$. In Fig. 10, it can be observed that the average number of iterations $N_{\text {Aver }}$ becomes larger when increasing $R_{D}$ and decreasing the operation $\mathrm{C} / \mathrm{N}$. By using the average number of iterations obtained in Fig. 10 and Eq. (45). Table 3 shows the ratio of computation complexity $(R)$ between the proposed TDE with the iterative method and the inverse matrix method. In the Table 3, it can be concluded that the proposed TDE with the iterative method can reduce the computation complexity to 0.078 (Approximately $7.8 \%)$ at $\mathrm{C} / \mathrm{N}$ is $14 \mathrm{~dB}$ and $R_{D}$ is $20 \%$ which corresponds to the vehicle speed $381 \mathrm{~km} / \mathrm{hrs}$. 


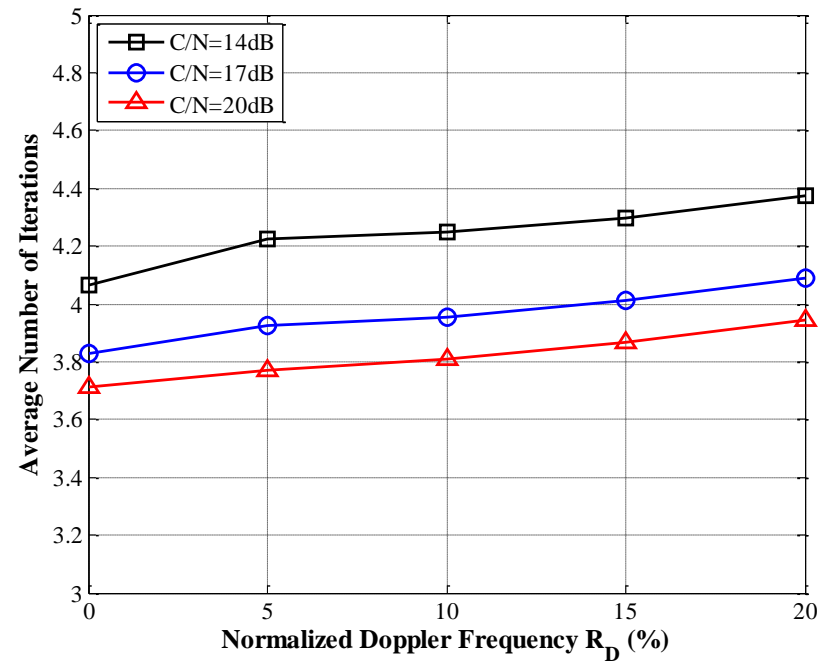

Figure. 10 An average number of required iterations for the proposed method when changing $R_{D}$ at $T O L=0.01$

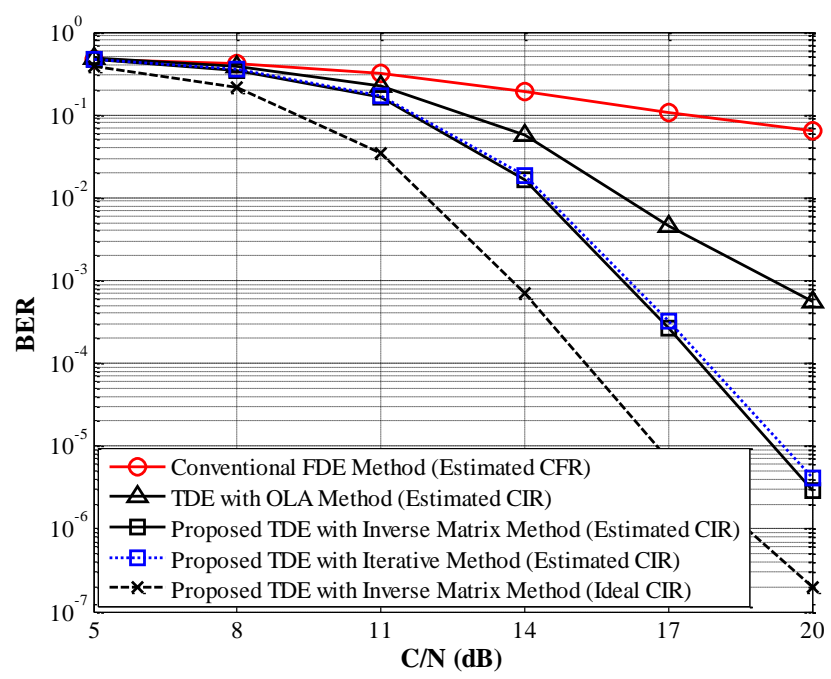

Figure. 11 BER performance for the proposed method when changing $\mathrm{C} / \mathrm{N}$ at $R_{D}=15 \%$

Fig. 11 shows the BER performances for the proposed TDE method both of the iterative and inverse matrix calculation methods when changing $\mathrm{C} / \mathrm{N}$ at $R_{D}=15 \%$ (Vehicle speed $286 \mathrm{~km} / \mathrm{hrs}$ ). In Fig. 11 , it can be observed that the proposed TDE method can achieve much better BER performance than the conventional FDE method and the TDE with OLA method [13] From the results in Fig. 11 and Table 3, it can be concluded that the proposed TDE with iterative method can achieve almost the same BER performance as the inverse matrix calculation method with much smaller computation complexity.

\section{Conclusions}

This paper proposes a low-complexity TDE method in conjunction with the time domain CIR estimation method. The salient features of the proposed method are to employ the time-domain training sequence (TS) in the CIR estimation and to employ the partial differentiation in solving the ML equation. By using partial differentiation, the coefficients matrix of simultaneous equations becomes the square matrix which can be solved iteratively without the inverse matrix calculation. From the verified computer simulation results, it can be concluded that the proposed TDE with the iterative method can achieve much better BER performance than the conventional FDE and TDE with OLA methods under higher time-varying fading channels(vehicle speed $\approx 381 \mathrm{~km} / \mathrm{hrs}$ ). The computation complexity for the proposed TDE with iterative method shows much lower than the inverse matrix method up to $90 \%$ with the same BER performance of inverse matrix calculation.

In future work, the proposed channel estimation and equalization methods could be applied to multiinput multiple-input multiple-output (MIMO) channels to increase the capacity by a factor of the minimum number of transmitting and receiving antennas.

\section{References}

[1] R. Prasad, OFDM for Wireless Communication Systems, Artech House, 2004.

[2] Y. G. Li and G. L. Stuber, Orthogonal Frequency Division Multiplexing for Wireless Communications, Springer, 2005.

[3] A. Goldsmith, Wireless Communications, Cambridge University Press, 2005.

[4] B. Farhang-Boroujeny and H. Moradi, "OFDM Inspired Waveforms for 5G”, IEEE Communications Surveys \& Tutorials, Vol. 18, No. 4, pp. 2474-2492, 2016.

[5] A. Rico-Alvarino, M. Vajapeyam, H. Xu, X. Wang, Y. Blankenship, J. Bergman, T. Tirronen, and E. Yavuz, "An overview of 3GPP enhancements on machine to machine communications", IEEE Communications Magazine, Vol. 54, No. 6, pp. 14-21, 2016.

[6] J. Ma, P. V. Orlik, J. Zhang and G. Y. Li, "Reduced-Rate OFDM Transmission for InterSubchannel Interference Self-Cancellation over High-Mobility Fading Channels", IEEE Trans., Vol. 11, No. 6, pp. 2013-2023, 2012.

[7] C. Y. Ma, S. W. Liu, and C. C. Huang, "LowComplexity ICI Suppression Methods Utilizing Cyclic Prefix for OFDM Systems in HighMobility Fading Channels", IEEE Transactions on Vehicular Technology, Vol. 63, No. 2, pp. 718-730, 2014.

[8] J. Wu and P. Fan, "A Survey on High Mobility Wireless Communications: Challenges, 
Opportunities and Solutions", IEEE Access, Vol. 4, pp. 450-476, 2016.

[9] G. Li, H. Yang, L. Cai, and L. Gui, "A Lowcomplexity Equalization Technique for OFDM System in Time-Variant Multipath Channels", In: Proc. of the 58th IEEE on Vehicular Technology Conference, Vol. 4, pp. 2466-2470, 2003.

[10] Y. Yu, S. Leung and F. Lu, "Iterative Frequency Domain Equalization for OFDM Over Doubly Selective Channels", In: Proc. of IEEE International Conference on Acoustics, Speech, and Signal Processing, Vol. 3, pp. III-453-III456, 2007.

[11] S. Ma, N. Wong, and T. S. Ng, "Time Domain Equalization for OFDM Systems", In: Proc. of IEEE International Symposium on Circuits and Systems, pp. 3946-3949, 2006.

[12] S. Takeuchi, K. Naito, K. Mori, and H. Kobayashi, "Proposal of Doppler Frequency Spread Compensation Method for OFDM Systems", In: Proc. of the 9th IEEE VTS Asia Pacific Wireless Communications Symposium, CO-5, 2012.

[13] P. Reangsuntea, P. Boonsrimuang, K. Mori, and H. Kobayashi, "Time Domain Equalization Method for TS-OFDM Signal under Higher Mobile Environments", EURASIP Journal on Wireless Communications and Networking, Vol. 2017, No. 5, pp. 1-12, 2017.

[14] T. Miyamoto, K. Naito, K. Mori, and H. Kobayashi, "Proposal of Doppler Estimation Method of Using Frequency Channel Response for OFDM Systems", In: Proc. of the 7th International Conference on Signal Processing and Communication Systems, 2013.

[15] G. Mkrtchyan, K. Naito, K. Mori, and H. Kobayashi, "ML Time Domain Channel Estimation and Equalization for OFDM without Guard Interval", In: Proc. of Electrical Engineering/Electronics, Computer, Telecommunication and Information Technology International Conference, pp. 473476, 2005.

[16] A. J. Viterbi, "Error Bounds for Convolutional Codes and an Asymptotically Optimum Decoding Algorithm", IEEE Transactions on Information Theory, Vol. IT-13, pp. 260-269, 1967.

[17] X. Yuan, Q. Guo, X. Wang, and L. Ping, "Evolution analysis of low-cost iterative equalization in coded linear systems with cyclic prefixes", IEEE J. Sel. Areas Commun., Vol. 26, No. 2, pp. 301-310, 2008.
[18] X. Cheng, Q. Yao, M. Wen, C. X. Wang, L. Y. Song, and B. L. Jiao, "Wideband Channel Modeling and Intercarrier Interference Cancellation for Vehicle-to-Vehicle Communication Systems", IEEE Journal on Selected Areas in Comm./Supplement, Vol. 31, No. 9, 2013.

[19] H. Kobayashi and K. Mori, "Proposal of Channel Estimation Method for OFDM Systems under Time-Varying Fading Environments", IEICE Technical Report, Vol.190-B, No.12, pp. 1249-1262, 2007.

[20] S. Coleri, M. Ergen, A. Puri and A. Bahai, "A Study of Channel Estimation in OFDM Systems", IEEE VTC2002-fall, 2002.

[21] C. Vulk, "Iterative Solution methods", Delft Institute of Applied Mathematics, 2012. 